\title{
Magnetic Neutral Line Rotations in Flare-Productive Regions
}

\author{
Takako T. Ishii, Ayumi Asai, Hiroki Kurokawa \\ Kwasan and Hida Observatories, Kyoto-U., Yamashina-ku, Kyoto, \\ JAPAN 607-8471 \\ Tsutomu T. Takeuchi \\ NAOJ, Mitaka, Tokyo, JAPAN 181-8588
}

\begin{abstract}
We examined the evolution of active regions and found that rotational motions of the neutral line in the $\delta$-type magnetic configuration are common in the flare-productive active regions.
\end{abstract}

\section{Introduction}

What is the common magnetic field configuration among flare-productive active regions? In our previous studies, we have found that the magnetic neutral line shows a rotational motion in a $\delta$-type active region NOAA 9026 , where three Xclass flares successively occurred (Kurokawa et al. 2002). In this paper, we show other examples of magnetic neutral line rotations in flare-productive regions.

\section{Results}

During the current solar maximum (cycle 23), we studied the evolution of all the active regions that have produced at least one X-class flare and have been observed by the Solar and Heliospheric Observatory (SOHO) / Michelson Doppler Imager (MDI). We examined 35 active regions from 1996 through 2003 June, and found clear rotational motions of the neutral line in the $\delta$-type magnetic configuration in the following active regions; NOAA 9026, 9393, 9415, 9591, $9661,9672,0039$, and 0314 . The angular velocity is about 20 degree par day in each active region and the motion continues about four days. The clockwise motion dominates in the north-hemisphere regions (three in three), and the counter-clock-wise in the south-hemisphere (three in five, one region shows both rotations). These motions suggest the existence of magnetic flux knots and its relation to magnetic helicity. Such a magnetic knot should be the energy storage for major flares.

\section{References}

Kurokawa, H., Wang, T.J., \& Ishii, T.T. 2002, ApJ, 572, 598 